\title{
CONICAL SQUARE FUNCTIONS AND NON-TANGENTIAL MAXIMAL FUNCTIONS WITH RESPECT TO THE GAUSSIAN MEASURE
}

\author{
Jan Maas, Jan van Neerven, and Pierre Portal
}

\begin{abstract}
We study, in $L^{1}\left(\mathbb{R}^{n} ; \gamma\right)$ with respect to the gaussian measure, nontangential maximal functions and conical square functions associated with the Ornstein-Uhlenbeck operator by developing a set of techniques which allow us, to some extent, to compensate for the non-doubling character of the gaussian measure. The main result asserts that conical square functions can be controlled in $L^{1}$-norm by non-tangential maximal functions. Along the way we prove a change of aperture result for the latter. This complements recent results on gaussian Hardy spaces due to Mauceri and Meda.
\end{abstract}

\section{Introduction}

Gaussian harmonic analysis, understood as the study of objects associated with the gaussian measure

$$
d \gamma(x)=(2 \pi)^{-n / 2} \exp \left(-\frac{1}{2}|x|^{2}\right) d x
$$

on $\mathbb{R}^{n}$, and the Ornstein-Uhlenbeck operator

$$
L f(x)=-\Delta f(x)+x \cdot \nabla f(x)
$$

on function spaces such as $L^{2}\left(\mathbb{R}^{n} ; \gamma\right)$, has recently gained new momentum following the development, by Mauceri and Meda [9], of an atomic Hardy space $H_{\mathrm{at}}^{1}\left(\mathbb{R}^{n} ; \gamma\right)$, on which various functions of $L$ give rise to bounded operators. Harmonic analysis in $L^{p}\left(\mathbb{R}^{n} ; \gamma\right)$ has been relatively well established for some time, with results such as the boundedness of Riesz

2010 Mathematics Subject Classification. Primary: 42B25; Secondary: 42B30, 60J35. Key words. Hardy spaces, Gaussian measure, Ornstein-Uhlenbeck operator, square function, maximal function.

The first named author is supported by Rubicon subsidy 680-50-0901 of the Netherlands Organisation for Scientific Research (NWO). The second named author is supported by VICI subsidy 639.033.604 of the Netherlands Organisation for Scientific Research (NWO). 
transforms going back to the work of Meyer and Pisier in the 1980's. The $p=1$ case, however, has always proven to be difficult. Over the last 30 years, some weak type $(1,1)$ estimates have been obtained, while others have been disproved (see the survey [13]). The proofs of these results rely on subtle decompositions and estimates of kernels. Until the seminal Mauceri-Meda paper appeared in 2007, a large part of euclidean harmonic analysis, such as end point estimates using Hardy and BMO spaces, seemed to have no gaussian counterpart. Gaussian harmonic analysis in $L^{2}\left(\mathbb{R}^{n} ; \gamma\right)$ is relatively straightforward given the fact that the Ornstein-Uhlenbeck operator is diagonal with respect to the basis of Hermite polynomials. The $L^{p}\left(\mathbb{R}^{n} ; \gamma\right)$ case, with $1<p<\infty$, is harder but still manageable through kernel estimates. The end points $p=1$ and $p=\infty$, however, usually require techniques such as Whitney coverings and Calderón-Zygmund decompositions, for which the non-doubling nature of the gaussian measure, has, so far, not been overcome. Mauceri and Meda's paper [9], though, indicates a possible strategy. The authors used the notion of admissible balls which goes back to the work of Muckenhoupt [11]. These are balls $B(x, r)$ with the property that $r \leq a \min \left(1, \frac{1}{|x|}\right)$ for some fixed admissibility parameter $a>0$. On these admissible balls, the gaussian measure turns out to be doubling. The idea is then to follow classical arguments using admissible balls only. This is easier said than done. Indeed, admissible balls are very small when their centre is far away from the origin, whereas tools such as Whitney decompositions of open sets require the size of balls to be comparable to their distance to the boundary of the set, hence possibly very large. This may be why, although it contains many breakthrough results, Mauceri and Meda's paper [9] does not yet give a full theory of $H^{1}$ and BMO spaces for the gaussian measure. For instance, the boundedness of key operators such as maximal functions, conical square functions (area integrals), and above all Riesz transforms, is still missing. In fact, while this paper was in its final stages, Mauceri, Meda, and Sjögren [10] proved that Riesz transforms (more precisely some Riesz transforms, see their paper for the details) are bounded from the Mauceri-Meda Hardy space $H_{\mathrm{at}}^{1}\left(\mathbb{R}^{n} ; \gamma\right)$ into $L^{1}\left(\mathbb{R}^{n} ; \gamma\right)$ only in dimension one. This suggests that the 'correct' gaussian Hardy space $H^{1}\left(\mathbb{R}^{n} ; \gamma\right)$ should be a modification of theirs.

In this paper, we take another step towards a satisfying $H^{1}\left(\mathbb{R}^{n} ; \gamma\right)$ theory by studying, in $L^{1}\left(\mathbb{R}^{n} ; \gamma\right)$, non-tangential square functions and maximal functions. These are gaussian analogues of the sublinear operators which, in the euclidean setting, are the cornerstones of the real 
variable theory of $H^{1}\left(\mathbb{R}^{n}\right)$. In the gaussian context, non-tangential maximal functions were first introduced in an unpublished work by Fabes and Forzani, who studied a gaussian counterpart of the Lusin area integral. Their $L^{p}$-boundedness was shown subsequently by Forzani, Scotto, and Urbina [6]. Our definition is an averaged version of a non-tangential maximal function from a subsequent paper of Pineda and Urbina [12]. The additional averaging adds some technical difficulties, but experience has shown (see e.g. [7]) that such averaging can be helpful in Hardy space theory and its applications (to boundary value problems, for instance).

Here we prove a change of aperture formula for the maximal function in the spirit of one of the key estimates of Coifman, Meyer, and Stein [3]. We then show that the non-tangential square function is controlled by the non-tangential maximal function. Such estimates are central in Hardy space theory (see for instance $[4],[5]$ ). Thus, the purpose of this article is twofold. On the one hand, it contributes to the development of dyadic techniques in gaussian harmonic analysis, i.e., methods and results based on gaussian analogues of the decomposition of $\mathbb{R}^{n}$ into dyadic cubes, the related covering lemmas, and the corresponding $H^{1}$ and weak type $(1,1)$ estimates. This makes the paper technical in nature, but we believe that the techniques developed here will find more applications, as gaussian harmonic analysis becomes more geometric and relies less on euclidean (after a change of variables) estimates of the Mehler kernel. On the other hand, this article gives some of the results required in the development of a gaussian Hardy space theory. When completed, such a theory will not only be satisfying from a pure harmonic analytic perspective, but it should also be applicable to stochastic partial differential equations (SPDE). Given the success of Hardy space techniques in deterministic PDE, one can think that a gaussian analogue would similarly have applications to non-linear SPDE and stochastic boundary value problems.

Now let us state the main result of this paper. We set

$$
m(x):=\min \left\{1, \frac{1}{|x|}\right\}
$$

and let

$$
\Gamma_{x}^{a}(\gamma):=\left\{(y, t) \in \mathbb{R}^{n} \times(0, \infty):|y-x|<t<a m(x)\right\}
$$

denote the admissible cone with parameter $a>0$ based at the point $x \in$ $\mathbb{R}^{n}$. We denote by $\left(e^{-t L}\right)_{t \geq 0}$ the Ornstein-Uhlenbeck semigroup acting on $L^{p}\left(\mathbb{R}^{n} ; \gamma\right)$ for $1<p<\infty$ (see the survey [13] and the references therein). For test functions $u \in C_{\mathrm{c}}\left(\mathbb{R}^{n}\right)$ and admissibility parameter $a>0$ 
we consider the conical square function

$$
S_{a} u(x)=\left(\int_{\Gamma_{x}^{a}(\gamma)} \frac{1}{\gamma(B(y, t))}\left|t \nabla e^{-t^{2} L} u(y)\right|^{2} d \gamma(y) \frac{d t}{t}\right)^{\frac{1}{2}},
$$

and the non-tangential maximal function

$$
T_{a}^{*} u(x):=\sup _{(y, t) \in \Gamma_{x}^{a}(\gamma)}\left(\frac{1}{\gamma(B(y, t))} \int_{B(y, t)}\left|e^{-t^{2} L} u(z)\right|^{2} d \gamma(z)\right)^{\frac{1}{2}} .
$$

The main result of this paper reads as follows:

Theorem 1.1. For each $a>0$ there exists an $a^{\prime}>0$ such that the conical square function $S_{a}$ is controlled by the non-tangential maximal function $T_{a^{\prime}}^{*}$, in the sense that

$$
\left\|S_{a} u\right\|_{L^{1}\left(\mathbb{R}^{n} ; \gamma\right)} \lesssim\left\|T_{a^{\prime}}^{*} u\right\|_{L^{1}\left(\mathbb{R}^{n} ; \gamma\right)}
$$

with implied constant independent of $u \in C_{\mathrm{c}}\left(\mathbb{R}^{n}\right)$.

By using the truncated cones $\Gamma_{x}^{a}$, we are only averaging over admissible balls in the definition of the operators. The idea is, of course, to exploit the doubling property of the gaussian measure on these balls. This makes the operators "admissible". The reader should notice, however, that they are not local, in the sense that their kernels are not supported in a region of the form $\left\{(x, y) \in \mathbb{R}^{2 n} ;|x-y| \leq \frac{a}{1+|x|+|y|}\right\}$. Moreover, they can not be written as sums of local operators. This is due to a lack of off-diagonal estimates, that is a crucial difference between the Ornstein-Uhlenbeck semigroup and the heat semigroup.

Acknowledgement. We are grateful to the anonymous referee for valuable suggestions that led to simplifications and generalisations of various arguments.

\section{A covering lemma}

In this section we introduce partitions of $\mathbb{R}^{n}$ into "admissible" dyadic cubes and use them to prove a covering lemma which will be needed later on.

We begin with a brief discussion of admissible balls. Let

$$
m(x):=\min \left\{1, \frac{1}{|x|}\right\}, \quad x \in \mathbb{R}^{n} .
$$

For $a>0$ we define

$$
\mathscr{B}_{a}:=\left\{B(x, r): x \in \mathbb{R}^{n}, \quad 0 \leq r \leq a m(x)\right\} .
$$


The balls in $\mathscr{B}_{a}$ are said to be admissible at scale $a$. It is a fundamental observation of Mauceri and Meda [9] that admissible balls enjoy a doubling property:

Lemma 2.1 (Doubling property). Let $a, \tau>0$. There exists a constant $d=d_{a, \tau, n}$, depending only on $a, \tau$, and the dimension $n$, such that if $B_{1}=B\left(c_{1}, r_{1}\right) \in \mathscr{B}_{a}$ and $B_{2}=B\left(c_{2}, r_{2}\right)$ have non-empty intersection and $r_{2} \leq \tau r_{1}$, then

$$
\gamma\left(B_{2}\right) \leq d \gamma\left(B_{1}\right)
$$

In particular this lemma implies that for all $a>0$ there exists a constant $d^{\prime}=d_{a, n}^{\prime}$ such that for all $B(x, r) \in \mathscr{B}_{a}$ we have

$$
\gamma(B(x, 2 r)) \leq d^{\prime} \gamma(B(x, r)) .
$$

The first part of the next lemma, which is taken from [8], says, among other things, that if $B(x, r) \in \mathscr{B}_{a}$ and $|x-y|<b r$, then $B(y, r) \in \mathscr{B}_{c}$ for some constant $c=c_{a, b}$ which depends only on $a$ and $b$.

Lemma 2.2. Let $a, b>0$ be given.

(i) If $r \leq a m(x)$ and $|x-y| \leq b r$, then $r \leq c_{a, b} m(y)$, where $c_{a, b}:=$ $a(1+a b)$.

(ii) If $|x-y| \leq b m(x)$, then $m(x) \leq(1+b) m(y)$ and $m(y) \leq(2+$ 2b) $m(x)$.

For $k \geq 0$ let $\Delta_{k}$ be the set of dyadic cubes at scale $k$, i.e.,

$$
\Delta_{k}=\left\{2^{-k}\left(x+[0,1)^{n}\right): x \in \mathbb{Z}^{n}\right\} .
$$

Following [8], in the gaussian case we only use cubes whose diameter depends on another parameter $l$, which keeps track of the distance from the ball to the origin. More precisely, define the layers

$$
L_{0}=[-1,1)^{n}, \quad L_{l}=\left[-2^{l}, 2^{l}\right)^{n} \backslash\left[-2^{l-1}, 2^{l-1}\right)^{n} \quad(l \geq 1),
$$

and define, for $k, l \geq 0$,

$$
\Delta_{k, l}^{\gamma}=\left\{Q \in \Delta_{l+k}: Q \subseteq L_{l}\right\}, \quad \Delta_{k}^{\gamma}=\bigcup_{l \geq 0} \Delta_{k, l}^{\gamma}, \quad \Delta^{\gamma}=\bigcup_{k \geq 0} \Delta_{k}^{\gamma} .
$$

Note that if $Q \in \Delta_{k}^{\gamma}$ with $Q \subseteq L_{l}$, then its centre $c_{Q}$ has norm $2^{l-1} \leq$ $\left|c_{Q}\right| \leq 2^{l} \sqrt{n}$ and we have

$$
\operatorname{diam}(Q)=2^{-k-l} \sqrt{n} \leq 2^{-k} n m\left(c_{Q}\right)
$$

Lemma 2.3. If a ball $B(x, r) \in \mathscr{B}_{a}$ intersects a cube $Q \in \Delta_{0}^{\gamma}$ with center $c_{Q}$, then

$$
r \leq 2 a(a+n) m\left(c_{Q}\right) .
$$


Proof: We consider two cases. First, if $\left|c_{Q}\right| \geq 2(a+n)$, we notice that $r \leq \frac{a}{|x|} \leq \frac{a}{\left|c_{Q}\right|-\left(r+n m\left(c_{Q}\right) / 2\right)} \leq \frac{a}{\left|c_{Q}\right|-(a+n / 2)} \leq \frac{2 a}{\left|c_{Q}\right|}=2 a m\left(c_{Q}\right) ;$ in the first inequality we used that $\operatorname{diam}(Q) \leq n m\left(c_{Q}\right)$ by $(2.1)$, in the second we used that $m\left(c_{Q}\right) \leq 1$ and $r \leq a m(x) \leq a$, the third follows from the assumption we made, and the final identity follows by noting that $\left|c_{Q}\right| \geq 2 n \geq 1$. Second, if $\left|c_{Q}\right| \leq 2(a+n)$, then together with $1 \leq$ $2(a+n)$ we obtain $1 \leq 2(a+n) m\left(c_{Q}\right)$ and $r \leq a \leq 2 a(a+n) m\left(c_{Q}\right)$.

We denote by $\alpha \circ Q$ the cube with the same centre as $Q$ and $\alpha$ times its side-length; similar notation is used for balls. Cubes in $\Delta^{\gamma}$ enjoy the following doubling property:

Lemma 2.4. Let $\alpha>0$. There exists a constant $C_{\alpha, n}$, depending only on $\alpha$ and the dimension $n$, such that for every cube $Q \in \Delta^{\gamma}$ we have

$$
\gamma(\alpha \circ Q) \leq C_{\alpha, n} \gamma(Q) \text {. }
$$

Proof: Without loss of generality we may assume that $\alpha>1$. Let $Q \in$ $\Delta_{k, l}^{\gamma}$ with center $y$ and side-length $2 s$. Set $B=B(y, s)$ and note that $B \subseteq Q$. Moreover, we have $\alpha \circ Q \subseteq \alpha \sqrt{n} \circ B$. Since, if $|y|>1$,

$$
2 s=\frac{\operatorname{diam}(Q)}{\sqrt{n}}=2^{-k-l} \leq 2^{-l} \leq \frac{\sqrt{n}}{|y|}=\sqrt{n} m(y)
$$

and, if $|y| \leq 1$

$$
2 s=2^{-k-l} \leq 1 \leq \sqrt{n} m(y)
$$

it follows that $B \in \mathscr{B}_{\sqrt{n} / 2}$. Using the doubling property for admissible balls from Lemma 2.1 we now obtain

$$
\gamma(\alpha \circ Q) \leq \gamma(\alpha \sqrt{n} \circ B) \leq C_{\alpha, n} \gamma(B) \leq C_{\alpha, n} \gamma(Q) .
$$

Lemma 2.5. Let $F \subseteq \mathbb{R}^{n}$ be a non-empty set, let $a, b, c>0$ be fixed, and let

$$
O_{a}:=\left\{x \in \mathbb{R}^{n}: 0<d(x, F) \leq a m(x)\right\} .
$$

There exists a sequence $\left(x_{k}\right)_{k \geq 1}$ in $O_{a}$ with the following properties:

(i) $O_{a} \subseteq \bigcup_{k \geq 1} B\left(x_{k}, b d\left(x_{k}, F\right)\right)$;

(ii) $\sum_{k \geq 1} \gamma\left(B\left(x_{k}, c d\left(x_{k}, F\right)\right)\right) \lesssim \gamma\left(O_{2 a}\right)$ with constant depending only on $a, b, c$, and $n$.

Remark 2.6. In the above lemma one actually has $\gamma\left(O_{2 a}\right) \lesssim \gamma\left(O_{a}\right)$, but since this fact is not needed below the rather technical proof is omitted. 
Proof: Let $\delta:=\min \left\{\frac{1}{2}, b\right\}$ and set $O:=O_{a}$ and $O^{\prime}:=O_{2 a}$ for brevity. We use a Whitney covering of $O^{\prime}$ by disjoint cubes $Q_{k}$ such that

$$
\frac{1}{4} \delta d\left(Q_{k}, \mathrm{CO}^{\prime}\right) \leq \operatorname{diam}\left(Q_{k}\right) \leq \delta d\left(Q_{k}, \boldsymbol{\complement} O^{\prime}\right),
$$

(see [14, VI.1]). We discard the cubes that do not intersect $O$ and relabel the remaining sequence of cubes as $\left(Q_{k}\right)_{k \geq 1}$ with centers $\left(c_{k}\right)_{k \geq 1}$. For each $k \geq 1$ pick $x_{k} \in O \cap Q_{k}$.

To check that the balls $B\left(c_{k}, \operatorname{diam}\left(Q_{k}\right)\right)$ are admissible, we use the fact that $\delta \leq \frac{1}{2}$ to obtain

$$
\left|c_{k}-x_{k}\right| \leq \frac{1}{2} \operatorname{diam}\left(Q_{k}\right) \leq \frac{1}{4} d\left(Q_{k}, \mathrm{CO}^{\prime}\right) \leq \frac{1}{4} d\left(x_{k}, F\right) \leq \frac{1}{4} a m\left(x_{k}\right) .
$$

Lemma 2.2(ii) then shows that $m\left(x_{k}\right) \leq\left(1+\frac{a}{4}\right) m\left(c_{k}\right)$. It follows that the balls $B\left(c_{k}, \operatorname{diam}\left(Q_{k}\right)\right)$ are admissible.

Next, $\operatorname{diam}\left(Q_{k}\right) \leq \delta d\left(Q_{k}, \complement O^{\prime}\right) \leq b d\left(x_{k}, F\right)$, so (i) follows from

$$
O \subseteq \bigcup_{k \geq 1} Q_{k} \subseteq \bigcup_{k \geq 1} B\left(x_{k}, \operatorname{diam}\left(Q_{k}\right)\right) \subseteq \bigcup_{k \geq 1} B\left(x_{k}, b d\left(x_{k}, F\right)\right) .
$$

Towards the proof of (ii), we claim that for all $x \in O$,

$$
d(x, F) \leq 3 \max \{1, a\} d\left(x, \mathrm{CO}^{\prime}\right) .
$$

To prove the claim, we fix $x \in O$ and pick an arbitrary $y \in \complement O^{\prime}$. Setting $\varepsilon:=\frac{1}{3} \min \left\{1, \frac{1}{a}\right\}$ we need to prove that

$$
|x-y| \geq \varepsilon d(x, F) .
$$

From $y \notin O^{\prime}$ we know that either $d(y, F) \geq 2 a m(y)$ or $d(y, F)=0$. In the latter case we have $y \in \bar{F}$, hence $\varepsilon d(x, F) \leq d(x, F) \leq|x-y|$, so in what follows we may assume that $d(y, F) \geq 2 a m(y)$. From $x \in O$ we know that $d(x, F) \leq a m(x)$. Suppose, for a contradiction, that $|x-y|<$ $\varepsilon d(x, F)$. Then $|x-y|<\varepsilon a m(x)$ and therefore $m(x) \leq(1+\varepsilon a) m(y)$ by Lemma 2.2(ii). Also, for all $f \in F$ we have $|x-y| \geq|y-f|-|f-x| \geq$ $2 a m(y)-|f-x|$. Minimising over $f$, this gives $|x-y| \geq 2 a m(y)-d(x, F)$. Since also $\varepsilon d(x, F)>|x-y|$, we find that $\operatorname{am}(y)<\frac{1}{2}(1+\varepsilon) d(x, F) \leq$ $\frac{1}{2}(1+\varepsilon) a m(x)$. It follows that $m(y)<\frac{1}{2}(1+\varepsilon) m(x)$, and in combination with the inequality $m(x) \leq(1+\varepsilon a) m(y)$ we get

$$
2<(1+\varepsilon)(1+\varepsilon a) .
$$

On the other hand, recalling that $\varepsilon=\frac{1}{3} \min \left\{1, \frac{1}{a}\right\}$ we see that $(1+\varepsilon)(1+$ $\varepsilon a) \leq\left(1+\frac{1}{3}\right)\left(1+\frac{1}{3}\right)=\frac{16}{9}<2$. This contradicts the previous inequality and the claim is proved.

Combining the estimate

$$
d\left(x_{k}, \mathrm{C} O^{\prime}\right) \leq d\left(Q_{k}, \mathrm{CO}^{\prime}\right)+\operatorname{diam}\left(Q_{k}\right) \leq\left(1+\frac{4}{\delta}\right) \operatorname{diam}\left(Q_{k}\right)
$$


with the claim, we obtain

$$
d\left(x_{k}, F\right) \leq 3 \max \{1, a\} d\left(x_{k}, \mathrm{CO}^{\prime}\right) \leq 3\left(1+\frac{4}{\delta}\right) \max \{1, a\} \operatorname{diam}\left(Q_{k}\right) .
$$

Recalling the inequality $\left|c_{k}-x_{k}\right| \leq \frac{1}{4} d\left(x_{k}, F\right)$ proved before, and then using the doubling property in combination with the above inequality, we obtain

$$
\begin{aligned}
\sum_{k \geq 1} \gamma\left(B\left(x_{k}, c d\left(x_{k}, F\right)\right)\right. & \leq \sum_{k \geq 1} \gamma\left(B\left(c_{k},\left(c+\frac{1}{4}\right) d\left(x_{k}, F\right)\right)\right. \\
& \lesssim \sum_{k \geq 1} \gamma\left(B\left(c_{k}, \operatorname{diam}\left(Q_{k}\right)\right) \lesssim \sum_{k \geq 1} \gamma\left(Q_{k}\right) \leq \gamma\left(O^{\prime}\right)\right.
\end{aligned}
$$

\section{Change of aperture for maximal functions}

In the proof of Theorem 1.1 we need a change of aperture result for the admissible cone appearing in the definition of non-tangential maximal functions. For this purpose we define, for $A, a>0$, the non-tangential maximal function with parameters $A, a$ by

$T_{(A, a)}^{*} u(x):=\sup _{(y, t) \in \Gamma_{x}^{(A, a)}(\gamma)}\left(\frac{1}{\gamma(B(y, A t))} \int_{B(y, A t)}\left|e^{-t^{2} L} u(z)\right|^{2} d \gamma(z)\right)^{\frac{1}{2}}$,

where

$$
\Gamma_{x}^{(A, a)}(\gamma):=\left\{(y, t) \in \mathbb{R}^{n} \times(0, \infty):|y-x|<A t \text { and } t<a m(x)\right\}
$$

is the admissible cone with parameters $A$, a based at the point $x \in \mathbb{R}^{n}$. The parameter $A$ is called the aperture of the cone.

In what follows we will fix the dimension $n$ and write $L^{p}(\gamma):=$ $L^{p}\left(\mathbb{R}^{n} ; \gamma\right)$.

Theorem 3.1 (Change of aperture). For all $A, A^{\prime}, a>0$ there exists a constant $D$, depending only on $A, A^{\prime}, a$, and the dimension $n$, such that for all $u \in L^{1}(\gamma)$ and $\sigma>0$ we have

$$
\gamma\left(\left\{x \in \mathbb{R}^{n}: T_{(A, a)}^{*} u(x)>D \sigma\right\}\right) \lesssim \gamma\left(\left\{x \in \mathbb{R}^{n}: T_{\left(A^{\prime}, a^{\prime}\right)}^{*} u(x)>\sigma\right\}\right)
$$

with $a^{\prime}=a(1+2 a A)\left(1+A^{\prime} a(1+2 a A)\right)$ and with implied constant independent of $u$ and $\sigma$. In particular,

$$
\left\|T_{(A, a)}^{*} u\right\|_{L^{1}(\gamma)} \lesssim\left\|T_{\left(A^{\prime}, a^{\prime}\right)}^{*} u\right\|_{L^{1}(\gamma)}
$$

with implied constant independent of $u \in L^{1}(\gamma)$. 
The proof of this theorem follows known arguments in the euclidean case [5]. We begin with a gaussian weak type $(1,1)$ estimate from $[\mathbf{9}]$. For the convenience of the reader we include an alternative and selfcontained proof.

Lemma 3.2. Let $a>0$. For $f \in L_{\text {loc }}^{1}\left(\mathbb{R}^{n}\right)$ put

$$
M_{a}^{*} f(x):=\sup _{B(x, r) \in \mathscr{B}_{a}} \frac{1}{\gamma(B(x, r))} \int_{B(x, r)}|f(y)| d \gamma(y) .
$$

Then for all $\tau>0$,

$$
\tau \gamma\left(\left\{M_{a}^{*}(f)>\tau\right\}\right) \lesssim\|f\|_{L^{1}(\gamma)}
$$

with implied constant only depending on a and $n$.

Proof: Fix $f \in L_{\text {loc }}^{1}\left(\mathbb{R}^{n}\right)$ and decompose it as $f=\sum_{Q \in \Delta_{0}^{\gamma}} 1_{Q} f$. We denote by $c_{Q}$ the centre of a cube $Q$. The idea of this proof is that the gaussian density is essentially equal to $e^{-\frac{1}{2}\left|c_{Q}\right|^{2}}$ on an admissible ball $B\left(c_{Q}, r_{Q}\right)$ and the support of $M_{A}^{*}\left(1_{Q} f\right)$ is included in such admissible balls.

To make this precise, consider a cube $Q \in \Delta_{0}^{\gamma}$, and suppose that a ball $B(x, r) \in \mathscr{B}_{a}$ intersects $Q$. Then Lemma 2.3 implies that $r \leq$ $2 a(a+n) m\left(c_{Q}\right)$. As a consequence, for any $y \in B(x, r)$, we use the triangle inequality and (2.1) to obtain

$$
\left|y-c_{Q}\right| \leq 2 r+\frac{1}{2} \operatorname{diam}(Q) \leq\left(4 a(a+n)+\frac{1}{2} n\right) m\left(c_{Q}\right)=: b_{a, n} m\left(c_{Q}\right),
$$

and thus

$\left.|| c_{Q}\right|^{2}-|y|^{2}|\leq| c_{Q}+y|| c_{Q}-y\left|\leq\left(2\left|c_{Q}\right|+\left|c_{Q}-y\right|\right)\right| c_{Q}-y \mid \leq 2 b_{a, n}+b_{a, n}^{2}$.

This inequality implies that

$$
e^{-\frac{1}{2}|y|^{2}} \approx e^{-\frac{1}{2}\left|c_{Q}\right|^{2}}
$$

with implied constants depending only on $a$ and $n$.

Using this estimate we obtain

(3.1) $\frac{1}{\gamma(B(x, r))} \int_{B(x, r)}\left|1_{Q}(y) f(y)\right| d \gamma(y) \lesssim \frac{1}{|B(x, r)|} \int_{B(x, r)}\left|1_{Q}(y) f(y)\right| d y$,

where $|B(x, r)|$ denotes the Lebesgue measure of the ball; the constants depend only on $a$ and $n$. 
Next we note that if $M_{a}^{*}\left(1_{Q} f\right)(x)>0$, then there exists a ball $B \in \mathscr{B}_{a}$ such that $B \cap Q \neq \emptyset$. Using (3.1), we thus have, for all $\tau>0$ and some $C>0$,

$$
\begin{aligned}
\gamma\left(\left\{M_{a}^{*}\left(1_{Q} f\right)>\tau\right\}\right) \approx \int_{\left\{M_{a}^{*}\left(1_{Q} f\right)>\tau\right\}} e^{-\frac{1}{2}|x|^{2}} d x & \lesssim e^{-\frac{1}{2}\left|c_{Q}\right|^{2}}\left|\left\{M_{a}^{*}\left(1_{Q} f\right)>\tau\right\}\right| \\
& \lesssim e^{-\frac{1}{2}\left|c_{Q}\right|^{2}}\left|\left\{M_{H L}\left(1_{Q} f\right)>C \tau\right\}\right|,
\end{aligned}
$$

where $M_{H L}$ denotes the euclidean Hardy-Littlewood maximal operator. Using the weak type $(1,1)$ bound for the latter, we get

$$
\tau \gamma\left(\left\{M_{a}^{*}\left(1_{Q} f\right)>\tau\right\}\right) \lesssim e^{-\frac{1}{2}\left|c_{Q}\right|^{2}} \int_{Q}|f(y)| d y \lesssim\left\|1_{Q} f\right\|_{L^{1}(\gamma)}
$$

with constants depending only on $a$ and $n$.

Now fix a cube $Q \in \Delta_{0}^{\gamma}$ with centre $c_{Q}$. For $x \in Q$ we have $\left|x-c_{Q}\right| \leq$ $\frac{1}{2} \operatorname{diam}(Q) \leq \frac{1}{2} n m\left(c_{Q}\right)$ by (2.1), and therefore by the second part of Lemma 2.2(ii), $m(x) \leq 2\left(1+\frac{1}{2} n\right) m\left(c_{Q}\right)$. Hence if $B(x, r) \in \mathcal{B}_{a}$ and $x \in Q$, then $r \leq \operatorname{am}(x) \leq(2+n) \operatorname{am}\left(c_{Q}\right)$. Thus

$$
B(x, r) \subseteq \bigcup_{l=1}^{N_{Q}} Q^{(l)}
$$

where we denote by $Q^{(l)}, l=1, \ldots, N_{Q}$, the cubes from $\Delta_{0}^{\gamma}$ that satisfy $d\left(Q, Q^{(l)}\right) \leq(2+n) a m\left(c_{Q}\right)$. Remark that $N_{Q} \leq N$, where $N=N_{a, n}$ only depends on $a$ and $n$.

It follows from the preceding considerations that

$$
M_{a}^{*} f(x) \leq M_{a}^{*}\left(\sum_{l=1}^{N_{Q}} 1_{Q^{(l)}} f\right)(x) \leq \sum_{l=1}^{N_{Q}} M_{a}^{*}\left(1_{Q^{(l)}} f\right)(x)
$$


for $x \in Q$, and thus

$$
\begin{aligned}
\gamma\left(\left\{M_{a}^{*} f>\tau\right\}\right) & =\sum_{Q \in \Delta_{0}^{\gamma}} \gamma\left(\left\{M_{a}^{*} f>\tau\right\} \cap Q\right) \\
& \leq \sum_{Q \in \Delta_{0}^{\gamma}} \gamma\left(\left\{\sum_{l=1}^{N_{Q}} M_{a}^{*}\left(1_{Q^{(l)}} f\right)>\tau\right\}\right) \\
& \leq \sum_{Q \in \Delta_{0}^{\gamma}} \sum_{l=1}^{N_{Q}} \gamma\left(\left\{M_{a}^{*}\left(1_{Q^{(l)}} f\right)>\frac{\tau}{N_{Q}}\right\}\right) \\
& \lesssim \sum_{Q \in \Delta_{0}^{\gamma}} \frac{N_{Q}}{\tau} \sum_{l=1}^{N_{Q}}\left\|1_{Q^{(l)}} f\right\|_{L^{1}(\gamma)} \\
& \lesssim \frac{N N^{\prime}}{\tau}\|f\|_{L^{1}(\gamma)},
\end{aligned}
$$

with implied constant depending only on $a$ and $n$; the $N^{\prime}$ in the last inequality accounts for the fact that, given $Q^{\prime} \in \Delta_{0}^{\gamma}$, there are at most $N^{\prime}$ cubes $Q \in \Delta_{0}^{\gamma}$ such that $\operatorname{dist}\left(Q, Q^{\prime}\right) \leq(2+n) a m\left(c_{Q}\right)$, where again $N^{\prime}$ depends only on $a$ and $n$.

Proof of Theorem 3.1: It suffices to prove the inequality for test functions $u \in C_{\mathrm{c}}\left(\mathbb{R}^{n}\right)$. For the rest of the proof we fix $u \in C_{\mathrm{c}}\left(\mathbb{R}^{n}\right)$. Using the doubling property on admissible balls, we fix a constant $\tau>0$ such that

$$
\gamma\left(B\left(y,\left(A^{\prime}+4 A\right) t\right)\right)<\frac{1}{\tau} \gamma\left(B\left(y, A^{\prime} t\right)\right) \quad \forall B(y, t) \in \mathscr{B}_{c_{a, 2 A}},
$$

where $c_{a, 2 A}=(1+2 a A) a$ is the constant arising from Lemma 2.2(i). For $\sigma>0$ we define

$$
\begin{aligned}
& E_{\sigma}:=\left\{x \in \mathbb{R}^{n}: T_{\left(A^{\prime}, a^{\prime}\right)}^{*} u(x)>\sigma\right\}, \\
& \widetilde{E_{\sigma}}:=\left\{x \in \mathbb{R}^{n}: M_{b}^{*}\left(1_{E_{\sigma}}\right)(x)>\tau\right\},
\end{aligned}
$$

where $M_{b}^{*} f$ is defined as in the lemma and $b:=\left(A^{\prime}+2 A\right) a$. The scheme of the proof is the following. We first prove (step 1) that, if $x \notin \widetilde{E}_{\sigma}$ and $(y, t) \in \Gamma_{x}^{(2 A, a)}(\gamma)$, then $B\left(y, A^{\prime} t\right) \nsubseteq E_{\sigma}$. We then use this fact (step 2) to prove that

$$
\frac{1}{\gamma\left(B\left(y, A^{\prime} t\right)\right)} \int_{B\left(y, A^{\prime} t\right)}\left|e^{-t^{2} L} u(\zeta)\right|^{2} d \gamma(\zeta) \leq \sigma^{2},
$$


for all $(y, t) \in \Gamma_{x}^{(2 A, a)}(\gamma)$ with $x \notin \widetilde{E_{\sigma}}$. This eventually gives (step 3) that there exists $D=D_{A, A^{\prime}, a, n}>0$ such that $\left\{x \in \mathbb{R}^{n} ; T_{(A, a)}^{*} u(x)>D \sigma\right\} \subseteq$ $\widetilde{E}_{\sigma}$. The proof is then concluded using Lemma 3.2 applied to $1_{E_{\sigma}}$. In the estimates that follow, the implicit constants are independent of $u$ and $\sigma$.

Throughout steps 1-3 below, we fix a point $x \notin \widetilde{E_{\sigma}}$ and a point $(y, t) \in \Gamma_{x}^{(2 A, a)}(\gamma)$

Step 1: We claim that $B\left(y, A^{\prime} t\right) \nsubseteq E_{\sigma}$. To prove this, first note that from $|x-y| \leq 2 A t$ we have

$$
B\left(y, A^{\prime} t\right) \subseteq B\left(x,\left(A^{\prime}+2 A\right) t\right) \subseteq B\left(y,\left(A^{\prime}+4 A\right) t\right) .
$$

Furthermore, $t \leq a m(x)$, and therefore $B\left(x,\left(A^{\prime}+2 A\right) t\right) \in \mathscr{B}_{\left(A^{\prime}+2 A\right) a}=$ $\mathscr{B}_{b}$. If we now assume that the claim is false, we get

$$
\begin{aligned}
M_{b}^{*}\left(1_{E_{\sigma}}\right)(x) & =\sup _{B(x, r) \in \mathscr{B}_{b}} \frac{\gamma\left(B(x, r) \cap E_{\sigma}\right)}{\gamma(B(x, r))} \\
& \geq \sup _{B(x, r) \in \mathscr{B}_{b}} \frac{\gamma\left(B(x, r) \cap B\left(y, A^{\prime} t\right)\right)}{\gamma(B(x, r))} \\
& \geq \frac{\gamma\left(B\left(x,\left(A^{\prime}+2 A\right) t\right) \cap B\left(y, A^{\prime} t\right)\right)}{\gamma\left(B\left(x,\left(A^{\prime}+2 A\right) t\right)\right)} \\
& =\frac{\gamma\left(B\left(y, A^{\prime} t\right)\right)}{\gamma\left(B\left(x,\left(A^{\prime}+2 A\right) t\right)\right)} \\
& \geq \frac{\gamma\left(B\left(y, A^{\prime} t\right)\right)}{\gamma\left(B\left(y,\left(A^{\prime}+4 A\right) t\right)\right)} \\
& >\tau
\end{aligned}
$$

where the second inequality uses that $B\left(x,\left(A^{\prime}+2 A\right) t\right) \in \mathscr{B}_{b}$ and the last one follows from the definition of the constant $\tau$ and the observation that $B(y, t) \in \mathscr{B}_{c_{a, 2 A}}$ by Lemma $2.2(\mathrm{i})$, using that $B(x, t) \in \mathscr{B}_{a}$ and $|x-y| \leq 2 A t$. This contradicts the fact that $x \notin \widetilde{E_{\sigma}}$ and the claim is proved.

Step 2: Since $B\left(y, A^{\prime} t\right) \nsubseteq E_{\sigma}$, there exists $\widetilde{y} \in B\left(y, A^{\prime} t\right)$ such that $\widetilde{y} \notin E_{\sigma}$, that is,

$$
\sup _{(z, s) \in \Gamma_{\tilde{y}}^{\left(A^{\prime}, a^{\prime}\right)}(\gamma)} \frac{1}{\gamma\left(B\left(z, A^{\prime} s\right)\right)} \int_{B\left(z, A^{\prime} s\right)}\left|e^{-s^{2} L} u(\zeta)\right|^{2} d \gamma(\zeta) \leq \sigma^{2} .
$$


Remark also that, since $B(y, t) \in \mathscr{B}_{c_{a, 2 A}}$ and thus $t \leq c_{a, 2 A} m(y)$, Lemma 2.2 implies that $t \leq a^{\prime} m(\widetilde{y})$. Thus $(y, t) \in \Gamma_{\widetilde{y}}^{\left(A^{\prime}, a^{\prime}\right)}(\gamma)$ and therefore (3.2) implies

$$
\frac{1}{\gamma\left(B\left(y, A^{\prime} t\right)\right)} \int_{B\left(y, A^{\prime} t\right)}\left|e^{-t^{2} L} u(\zeta)\right|^{2} d \gamma(\zeta) \leq \sigma^{2} .
$$

Step 3: Next let $(w, s) \in \Gamma_{x}^{(A, a)}(\gamma)$ be arbitrary and fixed for the moment. Then $w \in B(x, A s)$. For any $v \in B(w, A s)$ we have $|v-x| \leq|v-w|+$ $|w-x| \leq 2 A s$. Since also $s \leq a m(x)$, it follows that $(v, s) \in \Gamma_{x}^{(2 A, a)}(\gamma)$. Also, since $|v-w| \leq A s$ implies $B\left(v, A^{\prime} s\right) \subseteq B\left(w,\left(A^{\prime}+A\right) s\right)$, we have

$$
\gamma\left(B\left(v, A^{\prime} s\right)\right) \leq \gamma\left(B\left(w,\left(A^{\prime}+A\right) s\right)\right) \lesssim \gamma(B(w, A s))
$$

by the doubling property for admissible balls; the balls $B(w, A s)$ are indeed admissible by Lemma 2.2(i).

We can cover $B(w, A s)$ with finitely many balls of the form $B\left(v_{i}, A^{\prime} s\right)$ with $v_{i} \in B(w, A s)$; this can be achieved with $N=N\left(A, A^{\prime}, n\right)$ balls. We then have, by (3.3),

$$
\begin{aligned}
\frac{1}{\gamma(B(w, A s))} & \int_{B(w, A s)}\left|e^{-s^{2} L} u(z)\right|^{2} d \gamma(z) \\
& \lesssim \sum_{i=1}^{N} \frac{1}{\gamma\left(B\left(v_{i}, A^{\prime} s\right)\right)} \int_{B\left(v_{i}, A^{\prime} s\right)}\left|e^{-s^{2} L} u(z)\right|^{2} d \gamma(z) \lesssim \sigma^{2}
\end{aligned}
$$

Taking the supremum over all $(w, s) \in \Gamma_{x}^{(A, a)}(\gamma)$, we infer that there exists a constant $D>0$, depending only on $A, A^{\prime}, a$, and the dimension $n$, such that $T_{(A, a)}^{*} u(x) \leq D \sigma$ for all $x \notin \widetilde{E_{\sigma}}$.

We have now shown that $\left\{T_{(A, a)}^{*} u(x)>D \sigma\right\} \subseteq \widetilde{E_{\sigma}}$. The first assertion of the theorem follows from this via Lemma 3.2. The second assertion follows from the first by integration:

$$
\begin{aligned}
\left\|T_{(A, a)}^{*} u\right\|_{L^{1}(\gamma)} & =D \int_{0}^{\infty} \gamma\left(\left\{x \in \mathbb{R}^{n}: T_{(A, a)}^{*} u(x)>D \sigma\right\}\right) d \sigma \\
& \lesssim \int_{0}^{\infty} \gamma\left(\widetilde{E_{\sigma}}\right) d \sigma \lesssim \int_{0}^{\infty} \gamma\left(E_{\sigma}\right) d \sigma=\left\|T_{\left(A^{\prime}, a^{\prime}\right)}^{*} u\right\|_{L^{1}(\gamma)} .
\end{aligned}
$$

Since the choice of $A, A^{\prime}, a \geq 0$ was arbitrary, this concludes the proof. 


\section{Proof of Theorem 1.1}

In this section we follow the method pioneered in [5] for proving square function estimates in Hardy spaces. This method has recently been adapted in a variety of contexts (see $[\mathbf{1}],[\mathbf{2}],[\mathbf{7}]$ ). Here, we modify the version given in [7] to avoid using the doubling property on nonadmissible balls, and to take into account differences between the Laplace and the Ornstein-Uhlenbeck operators. As a typical example of the latter phenomenon, we start by proving a gaussian version of the parabolic Cacciopoli inequality. Recall that $L$ is the Ornstein-Uhlenbeck operator, defined for $f \in C_{\mathrm{b}}^{2}\left(\mathbb{R}^{n}\right)$ by

$$
L f(x)=-\Delta f(x)+x \cdot \nabla f(x) .
$$

Note that, for all $f, g \in C_{\mathrm{b}}^{2}\left(\mathbb{R}^{n}\right)$, one has the integration by parts formula

$$
\int_{\mathbb{R}^{n}} L f \cdot g d \gamma=\int_{\mathbb{R}^{n}} \nabla f \cdot \nabla g d \gamma
$$

Lemma 4.1. Let $v: \mathbb{R}^{n} \times(0, \infty) \rightarrow \mathbb{C}$ be a $C^{1,2}$-function such that $v(\cdot, t) \in C_{\mathrm{b}}^{2}\left(\mathbb{R}^{n}\right)$ for all $t>0$, and suppose that

$$
\partial_{t} v+L v=0
$$

on $I\left(x_{0}, t_{0}, 2 r\right):=B\left(x_{0}, 2 c r\right) \times\left[t_{0}-4 r^{2}, t_{0}+4 r^{2}\right]$ for some $r \in(0,1)$, $0<C_{0} \leq c \leq C_{1}<\infty$, and $t_{0}>4 r^{2}$. Then

$$
\int_{I\left(x_{0}, t_{0}, r\right)}|\nabla v(x, t)|^{2} d \gamma(x) d t \lesssim \frac{1+r\left|x_{0}\right|}{r^{2}} \int_{I\left(x_{0}, t_{0}, 2 r\right)}|v(x, t)|^{2} d \gamma(x) d t
$$

with implied constant depending only on the dimension $n, C_{0}$ and $C_{1}$.

Proof: Let $\eta \in C^{\infty}\left(\mathbb{R}^{n} \times(0, \infty)\right)$ be a cut-off function such that $0 \leq \eta \leq 1$ on $\mathbb{R}^{n} \times(0, \infty), \eta \equiv 1$ on $I\left(x_{0}, t_{0}, r\right), \eta \equiv 0$ on the complement of $I\left(x_{0}, t_{0}, 2 r\right)$, and

$$
\|\nabla \eta\|_{\infty} \lesssim \frac{1}{r}, \quad\left\|\partial_{t} \eta\right\|_{\infty} \lesssim \frac{1}{r^{2}}, \quad\|\Delta \eta\|_{\infty} \lesssim \frac{1}{r^{2}}
$$

with implied constants depending only on $n, C_{0}, C_{1}$. Then, in view of $\|x \cdot \nabla \eta\|_{\infty} \lesssim\left(\left|x_{0}\right|+2 r\right) \cdot \frac{1}{r}$ and recalling that $0<r<1$,

$$
\|L \eta\|_{\infty} \lesssim \frac{1}{r^{2}}+\frac{1}{r}\left|x_{0}\right|+1 \lesssim \frac{1+r\left|x_{0}\right|}{r^{2}},
$$

where the implied constants depend only on $n, C_{0}, C_{1}$. 
Considering real and imaginary parts separately, we may assume that all functions are real-valued. Integrating the identity

$$
(\eta \nabla v) \cdot(\eta \nabla v)=(v \nabla \eta-\nabla(v \eta)) \cdot(v \nabla \eta-\nabla(v \eta))
$$

and then using that

$$
\begin{aligned}
\int_{I\left(x_{0}, t_{0}, 2 r\right)} \eta^{2} \nabla(v \eta) \cdot \nabla(v \eta) d \gamma d t & \leq \int_{0}^{\infty} \int_{\mathbb{R}^{d}} \nabla(v \eta) \cdot \nabla(v \eta) d \gamma d t \\
& =\int_{0}^{\infty} \int_{\mathbb{R}^{n}} v \eta L(v \eta) d \gamma d t \\
& =\int_{I\left(x_{0}, t_{0}, 2 r\right)} v \eta L(v \eta) d \gamma d t
\end{aligned}
$$

we obtain

$$
\begin{aligned}
\int_{I\left(x_{0}, t_{0}, r\right)}|\nabla v|^{2} d \gamma d t & \leq \int_{I\left(x_{0}, t_{0}, 2 r\right)} \eta^{2}|\eta \nabla v|^{2} d \gamma d t \\
& \leq \int_{I\left(x_{0}, t_{0}, 2 r\right)} \eta^{2}|v \nabla \eta|^{2} d \gamma d t \\
& +\left|\int_{I\left(x_{0}, t_{0}, 2 r\right)} 2 v \eta^{2} \nabla(v \eta) \cdot \nabla \eta d \gamma d t\right| \\
& +\left|\int_{I\left(x_{0}, t_{0}, 2 r\right)} v \eta L(v \eta) d \gamma d t\right|
\end{aligned}
$$

For the first term on the right-hand side we have the estimate

$$
\int_{I\left(x_{0}, t_{0}, 2 r\right)} \eta^{2}|v \nabla \eta|^{2} d \gamma d t \lesssim \frac{1}{r^{2}} \int_{I\left(x_{0}, t_{0}, 2 r\right)}|v|^{2} d \gamma d t .
$$

For the second term we have, by (4.2),

$$
\begin{aligned}
\left|\int_{I\left(x_{0}, t_{0}, 2 r\right)} 2 v \eta^{2} \nabla(v \eta) \cdot \nabla \eta d \gamma d t\right| & =\frac{1}{2}\left|\int_{I\left(x_{0}, t_{0}, 2 r\right)} \nabla(v \eta)^{2} \cdot \nabla \eta^{2} d \gamma d t\right| \\
& \leq \frac{1}{2}\left|\int_{\mathbb{R}^{n}}(v \eta)^{2} L \eta^{2} d \gamma d t\right| \\
& \lesssim \frac{1+r\left|x_{0}\right|}{r^{2}} \int_{I\left(x_{0}, t_{0}, 2 r\right)}|v|^{2} d \gamma d t
\end{aligned}
$$


where we used the fact that $\eta^{2}$ satisfies the same assumptions as $\eta$ and (4.2) was applied to $\eta^{2}$. To estimate the third term on the right-hand side of (4.3) we substitute the identity

$$
L(v \eta)=\eta L v+v L \eta-2 \nabla v \cdot \nabla \eta=-\eta \partial_{t} v+v L \eta-2 \nabla v \cdot \nabla \eta
$$

and estimate each of the resulting integrals:

$$
\begin{aligned}
& \left|\int_{I\left(x_{0}, t_{0}, 2 r\right)} v \eta^{2} \partial_{t} v d \gamma d t\right|=\frac{1}{2}\left|\int_{I\left(x_{0}, t_{0}, 2 r\right)} \eta^{2} \partial_{t} v^{2} d \gamma d t\right| \\
& =\frac{1}{2}\left|\int_{I\left(x_{0}, t_{0}, 2 r\right)} v^{2} \partial_{t} \eta^{2} d \gamma d t\right| \\
& =\left|\int_{I\left(x_{0}, t_{0}, 2 r\right)} v^{2} \eta \partial_{t} \eta d \gamma d t\right| \\
& \lesssim \frac{1}{r^{2}} \int_{I\left(x_{0}, t_{0}, 2 r\right)}|v|^{2} d \gamma d t \\
& \left|\int_{I\left(x_{0}, t_{0}, 2 r\right)} v^{2} \eta L \eta d \gamma d t\right| \lesssim \frac{1+r\left|x_{0}\right|}{r^{2}} \int_{I\left(x_{0}, t_{0}, 2 r\right)}|v|^{2} d \gamma d t \\
& \left|\int_{I\left(x_{0}, t_{0}, 2 r\right)} v \eta \nabla v \cdot \nabla \eta d \gamma d t\right|=\frac{1}{4}\left|\int_{I\left(x_{0}, t_{0}, 2 r\right)} \nabla v^{2} \cdot \nabla \eta^{2} d \gamma d t\right| \\
& =\frac{1}{4}\left|\int_{\mathbb{R}^{n}} v^{2} L \eta^{2} d \gamma d t\right| \\
& \lesssim \frac{1+r\left|x_{0}\right|}{r^{2}} \int_{I\left(x_{0}, t_{0}, 2 r\right)}|v|^{2} d \gamma d t .
\end{aligned}
$$

Below we shall apply the lemma with $v(x, t)=e^{-t L} u(x)$, where $u$ is a function in $C_{\mathrm{c}}\left(\mathbb{R}^{n}\right)$. From the representation

$e^{-t L} u(x)=\int_{\mathbb{R}^{n}} M_{t}(x, y) u(y) d y$ where $M$ is the Mehler kernel (see, e.g., $[\mathbf{1 3}])$, it follows that $v$ satisfies the differentiability and boundedness assumptions of the lemma. 
We can now prove the main result of this paper. Recall that

$$
\begin{aligned}
S_{a} u(x) & =\left(\int_{\Gamma_{x}^{(1, a)}(\gamma)} \frac{1}{\gamma(B(y, t))}\left|t \nabla e^{-t^{2} L} u(y)\right|^{2} d \gamma(y) \frac{d t}{t}\right)^{\frac{1}{2}} \\
& =\left(\int_{\mathbb{R}^{n} \times(0, \infty)} \frac{1_{B(x, t)}(y)}{\gamma(B(y, t))} 1_{(0, a m(x))}(t)\left|t \nabla e^{-t^{2} L} u(y)\right|^{2} d \gamma(y) \frac{d t}{t}\right)^{\frac{1}{2}} .
\end{aligned}
$$

It will be convenient to define, for $\varepsilon>0$,

$$
S_{a}^{\varepsilon} u(x):=\left(\int_{\mathbb{R}^{n} \times(0, \infty)} \frac{1_{B(x, t)}(y)}{\gamma(B(y, t))} 1_{(\varepsilon, a m(x))}(t)\left|t \nabla e^{-t^{2} L} u(y)\right|^{2} d \gamma(y) \frac{d t}{t}\right)^{\frac{1}{2}}
$$

Proof of Theorem 1.1: As in the proof of Lemma 4.1 it suffices to consider real-valued $u \in C_{\mathrm{c}}\left(\mathbb{R}^{n}\right)$. Throughout the proof we fix $a>0$ and set $K:=c_{a, 1}$ and $\widetilde{K}:=c_{1+2 K, 2}$ using the notations of Lemma 2.2.

Let $F \subseteq \mathbb{R}^{n}$ be an arbitrary closed set and define

$$
F^{*}:=\left\{x \in \mathbb{R}^{n}: \gamma(F \cap B(x, r)) \geq \frac{1}{2} \gamma(B(x, r)) \quad \forall r \in(0, \widetilde{K} m(x)]\right\}
$$

Note that, since $F$ is closed, $F^{*} \subseteq F$. For $0<\varepsilon<1$ and $1<\alpha<2$ put

$R_{\alpha}^{\varepsilon}\left(F^{*}\right):=\left\{(y, t) \in \mathbb{R}^{n} \times(0, \infty): d\left(y, F^{*}\right)<\alpha t\right.$ and $\left.t \in\left(\alpha^{-1} \varepsilon, \alpha K m(y)\right)\right\}$

and let $\partial R_{\alpha}^{\varepsilon}\left(F^{*}\right)$ be its topological boundary. As in [5, p. 162] and $[\mathbf{1 4}$, p. 206] we may regularise this set and thus assume it admits a surface measure $d \sigma_{\alpha}^{\varepsilon}(y, t)$. Applying first Green's formula in $\mathbb{R}^{n}$ to the section of $R_{\alpha}^{\varepsilon}\left(F^{*}\right)$ at level $t$ and using the definition of $L$ (see (4.1)), and subsequently the fundamental theorem of calculus in the $t$-variable, we 
obtain the estimate

$$
\begin{aligned}
& \int_{F^{*}}\left|S_{a}^{\varepsilon} u(x)\right|^{2} d \gamma(x) \\
& =\int_{\mathbb{R}^{n} \times(0, \infty)} \int_{F^{*}} \frac{1_{B(x, t)}(y)}{\gamma(B(y, t))} 1_{(\varepsilon, a m(x))}(t)\left|t \nabla e^{-t^{2} L} u(y)\right|^{2} d \gamma(x) d \gamma(y) \frac{d t}{t} \\
& \stackrel{(\mathrm{i})}{\leq} \int_{\mathbb{R}^{n} \times(0, \infty)} \int_{F^{*}} \frac{1_{B(y, t)}(x)}{\gamma(B(y, t))} 1_{(\varepsilon, K m(y))}(t)\left|t \nabla e^{-t^{2} L} u(y)\right|^{2} d \gamma(x) d \gamma(y) \frac{d t}{t} \\
& \stackrel{(\mathrm{ii})}{=} \int_{\mathbb{R}^{n} \times(0, \infty)} \frac{\left.\gamma\left(B(y, t) \cap F^{*}\right)\right)}{\gamma(B(y, t))} 1_{\left\{d\left(y, F^{*}\right)<t\right\}} 1_{(\varepsilon, K m(y))}(t)\left|t \nabla e^{-t^{2} L} u(y)\right|^{2} d \gamma(y) \frac{d t}{t} \\
& \leq \int_{\mathbb{R}^{n} \times(0, \infty)} 1_{\left\{d\left(y, F^{*}\right)<t\right\}} 1_{(\varepsilon, K m(y))}(t)\left|t \nabla e^{-t^{2} L} u(y)\right|^{2} d \gamma(y) \frac{d t}{t} \\
& \leq \int_{R_{\alpha}^{\varepsilon}\left(F^{*}\right)}\left|t \nabla e^{-t^{2} L} u(y)\right|^{2} d \gamma(y) \frac{d t}{t} \\
& \lesssim \int_{R_{\alpha}^{\varepsilon}\left(F^{*}\right)} t L e^{-t^{2} L} u(y) \cdot e^{-t^{2} L} u(y) d \gamma(y) d t \\
& +\int_{\partial R_{\alpha}^{\varepsilon}\left(F^{*}\right)}\left|t \nabla e^{-t^{2} L} u \cdot \nu^{/ /}(y, t)\right|\left|e^{-t^{2} L} u(y)\right| e^{-\frac{1}{2}|y|^{2}} d \sigma_{\alpha}^{\varepsilon}(y, t) \\
& \lesssim \int_{R_{\alpha}^{\varepsilon}\left(F^{*}\right)}-\partial_{t}\left|e^{-t^{2} L} u(y)\right|^{2} d \gamma(y) d t \\
& +\int_{\partial R_{\alpha}^{\varepsilon}\left(F^{*}\right)}\left|t \nabla e^{-t^{2} L} u(y)\right|\left|e^{-t^{2} L} u(y)\right| e^{-\frac{1}{2}|y|^{2}} d \sigma_{\alpha}^{\varepsilon}(y, t) \\
& \lesssim \int_{\partial R_{\alpha}^{\varepsilon}\left(F^{*}\right)}\left|e^{-t^{2} L} u(y) \nu^{\perp}(y, t)\right|^{2} e^{-\frac{1}{2}|y|^{2}} d \sigma_{\alpha}^{\varepsilon}(y, t) \\
& +\int_{\partial R_{\alpha}^{\varepsilon}\left(F^{*}\right)}\left|t \nabla e^{-t^{2} L} u(y) \| e^{-t^{2} L} u(y)\right| e^{-\frac{1}{2}|y|^{2}} d \sigma_{\alpha}^{\varepsilon}(y, t) .
\end{aligned}
$$

In the above computation, $\nu / /$ denotes the projection of the normal vector $\nu$ to $R_{\alpha}^{\varepsilon}$ onto $\mathbb{R}^{n}$ and $\nu^{\perp}$ the projection of $\nu$ in the $t$ direction. In step (i) we used that $1_{B(x, t)}(y)=1_{B(y, t)}(x)$ and that $|x-y|<t$ and $t<a m(x)$ imply $t<K m(y)$ via Lemma 2.2(i); in step (ii) we used that $B(y, t) \cap F^{*} \neq \emptyset$ implies $d\left(y, F^{*}\right)<t$. Of course, all implied constants in the above inequalities are independent of $F, \varepsilon, \alpha$, and $u$. 
If $(y, t) \in \partial R_{\alpha}^{\varepsilon}\left(F^{*}\right)$, then either $d\left(y, F^{*}\right)=\alpha t$ and $t \in\left[\alpha^{-1} \varepsilon, \alpha K m(y)\right]$, or else $d\left(y, F^{*}\right)<\alpha t$ and $t \in\left\{\alpha^{-1} \varepsilon, \alpha K m(y)\right\}$. By examining these three cases separately, each time distinguishing between the possible relative positions of $m(y)$ with respect to the numbers $\frac{1}{2} \varepsilon, \alpha^{-1} \varepsilon$, and $\varepsilon$, one checks that $\partial R_{\alpha}^{\varepsilon}\left(F^{*}\right) \subseteq \widetilde{B}^{\varepsilon}:=\widetilde{B}_{1}^{\varepsilon} \cup \widetilde{B}_{2}^{\varepsilon} \cup \widetilde{B}_{3}^{\varepsilon}$ with

$$
\begin{aligned}
& \widetilde{B}_{1}^{\varepsilon}:=\left\{(y, t) \in \mathbb{R}^{n} \times(0, \infty): t \in\left[\frac{1}{2} \varepsilon, \min \{\varepsilon, m(y)\}\right] \text { and } d\left(y, F^{*}\right) \leq 2 t\right\}, \\
& \widetilde{B}_{2}^{\varepsilon}:=\left\{(y, t) \in \mathbb{R}^{n} \times(0, \infty): t \in[\varepsilon, m(y)] \text { and } t \leq d\left(y, F^{*}\right) \leq 2 t\right\}, \\
& \widetilde{B}_{3}^{\varepsilon}:=\left\{(y, t) \in \mathbb{R}^{n} \times(0, \infty): t \in[m(y), 2 K m(y)] \text { and } d\left(y, F^{*}\right) \leq 2 t\right\} .
\end{aligned}
$$

Now notice that, on $\partial R_{\alpha}^{\varepsilon}\left(F^{*}\right)$, we have either $t=\frac{\varepsilon}{\alpha}, t=\alpha K m(y)$, or $t=\alpha^{-1} d\left(y, F^{*}\right)$. Integrating over $\alpha \in(1,2)$ with respect to $\frac{d \alpha}{\alpha}$ and changing variables using that $\frac{d \alpha}{\alpha} \sim \frac{d t}{t}$, we obtain

$$
\begin{aligned}
\int_{F^{*}}\left|S_{a}^{\varepsilon} u\right|^{2} d \gamma \lesssim & \int_{\widetilde{B}^{\varepsilon}}\left|e^{-t^{2} L} u(y)\right|^{2} d \gamma(y) \frac{d t}{t} \\
& +\left(\int_{\widetilde{B}^{\varepsilon}}\left|e^{-t^{2} L} u(y)\right|^{2} d \gamma(y) \frac{d t}{t}\right)^{\frac{1}{2}}\left(\int_{\widetilde{B}^{\varepsilon}}\left|t \nabla e^{-t^{2} L} u(y)\right|^{2} d \gamma(y) \frac{d t}{t}\right)^{\frac{1}{2}} \\
& \lesssim \int_{\widetilde{B}^{\varepsilon}}\left|e^{-t^{2} L} u(y)\right|^{2} d \gamma(y) \frac{d t}{t}+\int_{\widetilde{B}^{\varepsilon}}\left|t \nabla e^{-t^{2} L} u(y)\right|^{2} d \gamma(y) \frac{d t}{t} .
\end{aligned}
$$

Here, and in the estimates to follow, the implied constants are independent of $F, \varepsilon$, and $u$.

We have to estimate the following six integrals:

$$
\begin{aligned}
& I_{1}:=\int_{\widetilde{B}_{1}^{\varepsilon}}\left|e^{-t^{2} L} u(y)\right|^{2} d \gamma(y) \frac{d t}{t}, \quad I_{2}:=\int_{\widetilde{B}_{1}^{\varepsilon}}\left|t \nabla e^{-t^{2} L} u(y)\right|^{2} d \gamma(y) \frac{d t}{t}, \\
& I_{3}:=\int_{\widetilde{B}_{2}^{\varepsilon}}\left|e^{-t^{2} L} u(y)\right|^{2} d \gamma(y) \frac{d t}{t}, \quad I_{4}:=\int_{\widetilde{B}_{2}^{\varepsilon}}\left|t \nabla e^{-t^{2} L} u(y)\right|^{2} d \gamma(y) \frac{d t}{t}, \\
& I_{5}:=\int_{\widetilde{B}_{3}^{\varepsilon}}\left|e^{-t^{2} L} u(y)\right|^{2} d \gamma(y) \frac{d t}{t}, \quad I_{6}:=\int_{\widetilde{B}_{3}^{\varepsilon}}\left|t \nabla e^{-t^{2} L} u(y)\right|^{2} d \gamma(y) \frac{d t}{t} .
\end{aligned}
$$

We start with $I_{1}$ and remark that, for $(y, t) \in \widetilde{B}_{1}^{\varepsilon}$, there exists $x \in F^{*}$ such that $|x-y| \leq 2 t$. Since $t \leq \min \{\varepsilon, m(y)\} \leq m(y)$, by Lemma 2.2(i) we have $t \leq c_{1,2} m(x)$ and hence $t \leq \widetilde{K} m(x)$, noting that $c_{1,2} \leq c_{1+2 K, 2}=$ $\widetilde{K}$. Therefore, by the definition of $F^{*}$,

$$
\gamma(F \cap B(x, t)) \geq \frac{1}{2} \gamma(B(x, t)) .
$$


(At this point the reader may wonder why $F^{*}$ is defined in terms of $\widetilde{K}$ and not in terms of $c_{1,2}$. The reason is that the argument will be repeated in the estimation of $I_{2}, I_{5}$, and $I_{6}$; in the latter two cases, the definition of $B_{3}^{\varepsilon}$ implies that one only gets $t \leq 2 K m(y)$ and hence $t \leq c_{2 K, 2} m(x) \leq$ $c_{1+2 K, 2} m(x)$.) By (4.4) and doubling property for the admissible ball $B(x, t) \in \mathscr{B}_{c_{1,2}}$,

$\gamma(F \cap B(y, 3 t)) \geq \gamma(F \cap B(x, t)) \geq \frac{1}{2} \gamma(B(x, t)) \gtrsim \gamma(B(x, 3 t)) \geq \gamma(B(y, t))$,

and therefore, writing $d:=c_{1,3}$,

$$
\begin{aligned}
I_{1} & \lesssim \int_{\widetilde{B}_{1}^{\varepsilon}} \int_{F \cap B(y, 3 t)} \frac{1}{\gamma(B(y, t))}\left|e^{-t^{2} L} u(y)\right|^{2} d \gamma(z) d \gamma(y) \frac{d t}{t} \\
& \leq \int_{\mathbb{R}^{n}} \int_{\frac{1}{2} \varepsilon}^{\frac{1}{2} \varepsilon \vee \min \{\varepsilon, m(y)\}} \int_{F} \frac{1_{B(y, 3 t)}(z)}{\gamma(B(y, t))}\left|e^{-t^{2} L} u(y)\right|^{2} d \gamma(z) \frac{d t}{t} d \gamma(y) \\
& \leq \int_{F} \int_{\frac{1}{2} \varepsilon}^{\frac{1}{2} \varepsilon \vee \min \{\varepsilon, d m(z)\}} \int_{B(z, 3 t)} \frac{1}{\gamma(B(y, t))}\left|e^{-t^{2} L} u(y)\right|^{2} d \gamma(y) \frac{d t}{t} d \gamma(z),
\end{aligned}
$$

where in the last inequality we used that $t \leq m(y)$ and $|y-z|<3 t$ imply $t \leq d m(z)$ by Lemma $2.2(\mathrm{i})$.

Fix $(z, t) \in F \times\left(\frac{1}{2} \varepsilon, \frac{1}{2} \varepsilon \vee \min \{\varepsilon, d m(z)\}\right)$. For all $y \in B(z, 3 t)$ we have $B(z, 3 t) \subseteq B(y, 6 t)$ and therefore, by the doubling property for $B(y, t)$ (noting that from $t<d m(z)$ and $|z-y|<3 t$ it follows that $t<\widetilde{d} m(y)$ where $\widetilde{d}:=c_{d, 3}$, so $B(y, t)$ is an admissible ball in $\left.\mathscr{B}_{\widetilde{d}}\right)$,

$$
\begin{aligned}
\int_{B(z, 3 t)} \frac{1}{\gamma(B(y, t))}\left|e^{-t^{2} L} u(y)\right|^{2} d \gamma(y) & \lesssim \frac{1}{\gamma(B(z, 3 t))} \int_{B(z, 3 t)}\left|e^{-t^{2} L} u(y)\right|^{2} d \gamma(y) \\
& \leq\left|T_{(3, d)}^{*} u(z)\right|^{2}
\end{aligned}
$$

where the last inequality follows from $(z, t) \in \Gamma_{z}^{(3, d)}(\gamma)$. Combining this with the previous inequality it follows that

$$
I_{1} \lesssim \int_{F} \int_{\frac{1}{2} \varepsilon}^{\varepsilon}\left|T_{(3, d)}^{*} u(z)\right|^{2} \frac{d t}{t} d \gamma(z) \lesssim \int_{F}\left|T_{(3, d)}^{*} u(z)\right|^{2} d \gamma(z)
$$


We proceed similarly for $I_{2}$, using Lemma 4.1 to handle the gradient. With $\tau(z):=d m(z)$ we have, proceeding as in (4.5),

$$
\begin{aligned}
I_{2} & \lesssim \int_{F} \int_{\frac{1}{2} \varepsilon}^{\frac{1}{2} \varepsilon \vee \min \{\varepsilon, \tau(z)\}} \int_{B(z, 3 t)} \frac{1}{\gamma(B(y, t))}\left|t \nabla e^{-t^{2} L} u(y)\right|^{2} d \gamma(y) \frac{d t}{t} d \gamma(z) \\
& \stackrel{(\mathrm{i})}{\lesssim} \int_{F \cap\left\{\tau(z) \geq \frac{1}{2} \varepsilon\right\}} \int_{\frac{1}{2} \varepsilon}^{\varepsilon} \frac{1}{\gamma(B(z, 3 \varepsilon))} \int_{B(z, 3 \varepsilon)}\left|t \nabla e^{-t^{2} L} u(y)\right|^{2} d \gamma(y) \frac{d t}{t} d \gamma(z) \\
& \stackrel{(\mathrm{ii})}{\lesssim} \int_{F \cap\left\{\tau(z) \geq \frac{1}{2} \varepsilon\right\}} \sum_{l=2}^{7} \int_{\frac{l \varepsilon^{2}}{8}}^{\frac{(l+1) \varepsilon^{2}}{8}} \frac{1}{\gamma(B(z, 3 \varepsilon))} \int_{B(z, 3 \varepsilon)}\left|\nabla e^{-s L} u(y)\right|^{2} d \gamma(y) d s d \gamma(z) .
\end{aligned}
$$

In (i) we used the inclusions $B(z, 3 t) \subseteq B(z, 3 \varepsilon) \subseteq B(z, 6 t) \subseteq B(y, 9 t)$ together with the doubling property for $B(y, t)$, and in (ii) we substituted $t^{2}=s$.

For each $l \in\{2, \ldots, 7\}$ we apply Lemma 4.1 with $t_{0}^{l}=\frac{1}{2}\left(\frac{l \varepsilon^{2}}{8}+\right.$ $\left.\frac{(l+1) \varepsilon^{2}}{8}\right)=\frac{(2 l+1) \varepsilon^{2}}{16}, c^{l}=12$ and $\left(r^{l}\right)^{2}=\frac{\varepsilon^{2}}{16}$. Together with the doubling property for $B(z, \varepsilon)$ (noting that $B(z, \varepsilon) \in \mathscr{B}_{2 d}$ in view of $\varepsilon \leq 2 t \leq$ $2 d m(z))$, this gives

$$
\begin{aligned}
I_{2} \lesssim \int_{F \cap\left\{\tau(z) \geq \frac{1}{2} \varepsilon\right\}} & \sum_{l=2}^{7} \int_{\frac{(2 l-3) \varepsilon^{2}}{16}}^{\frac{(2 l+5) \varepsilon^{2}}{16}} \frac{1+r^{l}|z|}{\left(r^{l}\right)^{2}} \\
& \times \frac{1}{\gamma(B(z, 6 \varepsilon))} \int_{B(z, 6 \varepsilon)}\left|e^{-s L} u(y)\right|^{2} d \gamma(y) d s d \gamma(z) .
\end{aligned}
$$

Fix $(z, s) \in\left(F \cap\left\{\tau(z) \geq \frac{1}{2} \varepsilon\right\}\right) \times\left(\frac{1}{16} \varepsilon^{2}, \frac{19}{16} \varepsilon^{2}\right)$. Then from $B(z, 6 \varepsilon) \subseteq$ $B(z, 24 \sqrt{s}) \subseteq B(z, 30 \varepsilon)$ and the doubling property for the balls $B(z, \varepsilon) \in$ $\mathscr{B}_{2 d}$ (note that $\left.\varepsilon \leq 2 \tau(z)=2 d m(z)\right)$,

$$
\begin{aligned}
& \frac{1}{\gamma(B(z, 6 \varepsilon))} \int_{B(z, 6 \varepsilon)}\left|e^{-s L} u(y)\right|^{2} d \gamma(y) \\
& \quad \lesssim \frac{1}{\gamma(B(z, 24 \sqrt{s}))} \int_{B(z, 24 \sqrt{s})}\left|e^{-s L} u(y)\right|^{2} d \gamma(y) \leq\left|T_{(24,4 d)}^{*} u(z)\right|^{2}
\end{aligned}
$$


where the last step follows from $(z, \sqrt{s}) \in \Gamma_{z}^{(24,4 d)}(\gamma)$. Combining this with the previous estimate we obtain

$$
\begin{aligned}
I_{2} & \lesssim \int_{F} \sum_{l=2}^{7} \int_{\frac{(2 l-3) \varepsilon^{2}}{16}}^{\frac{(2 l+5) \varepsilon^{2}}{16}} \frac{1+r^{l}|z|}{\left(r^{l}\right)^{2}}\left|T_{(24,4 d)}^{*} u(z)\right|^{2} d s d \gamma(z) \\
& \lesssim \int_{F}(1+\varepsilon|z|)\left|T_{(24,4 d)}^{*} u(z)\right|^{2} d \gamma(z),
\end{aligned}
$$

where the last step follows from the fact that $r^{l}=\frac{1}{4} \varepsilon$.

We proceed with an estimate for $I_{3}$. For $a>0$ let

$$
G_{a}:=\left\{y \in \mathbb{R}^{n}: 0<d\left(y, F^{*}\right) \leq a m(y)\right\} .
$$

Using Lemma 2.5, we cover $G_{2}$ with a sequence of balls $B\left(x_{k}, r_{k}\right)$ with $x_{k} \in G_{2}$ and $r_{k}=\frac{1}{4} d\left(x_{k}, F^{*}\right)$ for all $k$, and

$$
\sum_{k \geq 1} \gamma\left(B\left(x_{k}, d\left(x_{k}, F^{*}\right)\right)\right) \lesssim \gamma\left(G_{4}\right) \leq \gamma\left(\complement F^{*}\right),
$$

with implied constant independent of $u$ and $F$. Note that $B\left(x_{k}, r_{k}\right) \in$ $\mathscr{B}_{\frac{1}{2}}$ for all $k$.

If $(y, t) \in \widetilde{B}_{2}^{\varepsilon}$, then $y \in G_{2}$ and therefore $y \in B\left(x_{k}, r_{k}\right)$ for some $k$, and $\frac{1}{2} d\left(y, F^{*}\right) \leq t \leq d\left(y, F^{*}\right)$. It follows that

$$
\begin{aligned}
I_{3} & \leq \sum_{k} \int_{B\left(x_{k}, r_{k}\right)} \int_{\frac{1}{2} d\left(y, F^{*}\right)}^{d\left(y, F^{*}\right)}\left|e^{-t^{2} L} u(y)\right|^{2} \frac{d t}{t} d \gamma(y) \\
& \leq \sum_{k} \int_{B\left(x_{k}, r_{k}\right)} \int_{\frac{1}{4} d\left(x_{k}, F^{*}\right)}^{\frac{5}{4} d\left(x_{k}, F^{*}\right)}\left|e^{-t^{2} L} u(y)\right|^{2} \frac{d t}{t} d \gamma(y) \\
& \leq \sum_{k} \int_{\frac{1}{4} d\left(x_{k}, F^{*}\right)}^{\frac{5}{4} d\left(x_{k}, F^{*}\right)} \int_{B\left(x_{k}, t\right)}\left|e^{-t^{2} L} u(y)\right|^{2} d \gamma(y) \frac{d t}{t} .
\end{aligned}
$$

In the second inequality we used that $y \in B\left(x_{k}, r_{k}\right)$ implies $\left|x_{k}-y\right|<$ $r_{k}=\frac{1}{4} d\left(x_{k}, F^{*}\right)$, and the third inequality follows from Fubini's theorem and the inequality $r_{k}=\frac{1}{4} d\left(x_{k}, F^{*}\right) \leq \frac{1}{2} d\left(y, F^{*}\right) \leq t$.

Fix an index $k$ and a number $t \in\left(\frac{1}{4} d\left(x_{k}, F^{*}\right), \frac{5}{4} d\left(x_{k}, F^{*}\right)\right)$. Since $F^{*}$ is contained in the closure of $F$ we may pick $z_{k} \in F$ such that $\left|x_{k}-z_{k}\right|<2 d\left(x_{k}, F^{*}\right)$. By the choice of $t$ this implies $\left|x_{k}-z_{k}\right|<8 t$. Since by assumption we have $t \leq \frac{5}{4} d\left(x_{k}, F^{*}\right) \leq \frac{5}{2} m\left(x_{k}\right)$ (the second inequality being a consequence of $\left.x_{k} \in G_{2}\right)$, and since $\left|x_{k}-z_{k}\right|<8 t$, from Lemma 2.2 we conclude that $t \leq d m\left(z_{k}\right)$ with $d:=c_{\frac{5}{2}, 8}$. We 
conclude that $\left(x_{k}, t\right) \in \Gamma_{z_{k}}^{(8, d)}(\gamma)$ (since by definition this means that $\left.\left|x_{k}-z_{k}\right| \leq 8 t \leq 8 d m\left(z_{k}\right)\right)$ and consequently, using the doubling property for the admissible ball $B\left(x_{k}, t\right) \in \mathscr{B}_{\frac{5}{2}}$,

$$
\begin{aligned}
& \frac{1}{\gamma\left(B\left(x_{k}, t\right)\right)} \int_{B\left(x_{k}, t\right)}\left|e^{-t^{2} L} u(y)\right|^{2} d \gamma(y) \\
& \quad \lesssim \frac{1}{\gamma\left(B\left(x_{k}, 8 t\right)\right)} \int_{B\left(x_{k}, 8 t\right)}\left|e^{-t^{2} L} u(y)\right|^{2} d \gamma(y) \leq\left|T_{(8, d)}^{*} u\left(z_{k}\right)\right|^{2} .
\end{aligned}
$$

Combining this with the previous inequalities we obtain

$$
\begin{aligned}
I_{3} & \lesssim\left(\sup _{z \in F}\left|T_{(8, d)}^{*} u(z)\right|^{2}\right) \sum_{k} \int_{\frac{1}{4} d\left(x_{k}, F^{*}\right)}^{\frac{5}{4} d\left(x_{k}, F^{*}\right)} \gamma\left(B\left(x_{k}, t\right)\right) \frac{d t}{t} \\
& \lesssim\left(\sup _{z \in F}\left|T_{(8, d)}^{*} u(z)\right|^{2}\right) \sum_{k} \gamma\left(B\left(x_{k}, \frac{5}{4} d\left(x_{k}, F^{*}\right)\right)\right) \\
& \lesssim\left(\sup _{z \in F}\left|T_{(8, d)}^{*} u(z)\right|^{2}\right) \gamma\left(\complement F^{*}\right),
\end{aligned}
$$

where the last step used (4.6) and the doubling property (recall that $d\left(x_{k}, F^{*}\right) \leq 2 m\left(x_{k}\right)$, so the balls $B\left(x_{k}, d\left(x_{k}, F^{*}\right)\right)$ belong to $\left.\mathscr{B}_{2}\right)$.

To estimate $I_{4}$, we let $G_{2}$ and $B\left(x_{k}, r_{k}\right)$ be as in the previous estimate. Proceeding as in the first two lines of (4.7) and applying the Fubini theorem, we get

$$
\begin{aligned}
I_{4} & \lesssim \sum_{k} \int_{\frac{1}{4} d\left(x_{k}, F^{*}\right)}^{\frac{5}{4} d\left(x_{k}, F^{*}\right)} \int_{B\left(x_{k}, r_{k}\right)}\left|t \nabla e^{-t^{2} L} u(y)\right|^{2} d \gamma(y) \frac{d t}{t} \\
& =\frac{1}{2} \sum_{k} \sum_{l=2}^{49} \int_{\frac{2 l}{64} d^{2}\left(x_{k}, F^{*}\right)}^{\frac{2 l+2}{64} d^{2}\left(x_{k}, F^{*}\right)} \int_{B\left(x_{k}, r_{k}\right)}\left|\nabla e^{-s L} u(y)\right|^{2} d \gamma(y) d s .
\end{aligned}
$$

By Lemma 4.1, applied with $t_{0}=\frac{2 l+1}{64} d^{2}\left(x_{k}, F^{*}\right), c=2$ and $r=\frac{1}{8} d\left(x_{k}, F^{*}\right)$, this gives the estimate

$$
\begin{aligned}
I_{4} \lesssim \sum_{k} \sum_{l=2}^{49} \int_{\frac{2 l-3}{64} d^{2}\left(x_{k}, F^{*}\right)}^{\frac{2 l+5}{64} d^{2}\left(x_{k}, F^{*}\right)} \frac{1+d\left(x_{k}, F^{*}\right)\left|x_{k}\right|}{d^{2}\left(x_{k}, F^{*}\right)} \\
\times \int_{B\left(x_{k}, \frac{1}{2} d\left(x_{k}, F^{*}\right)\right)}\left|e^{-s L} u(y)\right|^{2} d \gamma(y) d s \\
\leq \sum_{k} \sum_{l=2}^{49} \int_{\frac{2 l-3}{64} d^{2}\left(x_{k}, F^{*}\right)}^{\frac{2 l+5}{64} d^{2}\left(x_{k}, F^{*}\right)} \frac{3}{d^{2}\left(x_{k}, F^{*}\right)} \int_{B\left(x_{k}, 4 \sqrt{s}\right)}\left|e^{-s L} u(y)\right|^{2} d \gamma(y) d s,
\end{aligned}
$$


where we used that $d\left(x_{k}, F^{*}\right) \leq 2 m\left(x_{k}\right) \leq \frac{2}{\left|x_{k}\right|}$ and that $s \geq \frac{1}{64} d^{2}\left(x_{k}, F^{*}\right)$ implies $\frac{1}{2} d\left(x_{k}, F^{*}\right) \leq 4 \sqrt{s}$.

Fix $k$ and pick an element $z_{k} \in F$ such that $\left|x_{k}-z_{k}\right|<2 d\left(x_{k}, F^{*}\right)$. Then for all $s$ in the range of integration we have $\left|x_{k}-z_{k}\right|<16 \sqrt{s}$. Since $\sqrt{s} \leq \frac{3}{2} d\left(x_{k}, F^{*}\right) \leq 3 m\left(x_{k}\right)$, from Lemma 2.2 we conclude that $\sqrt{s} \leq d m\left(z_{k}\right)$ with $d:=c_{3,16}$. We conclude that $\left(x_{k}, 4 \sqrt{s}\right) \in \Gamma_{z_{k}}^{(4,4 d)}(\gamma)$. This gives

$$
\begin{aligned}
I_{4} & \lesssim\left(\sup _{z \in F}\left|T_{(4,4 d)}^{*} u(z)\right|^{2}\right) \sum_{k} \frac{1}{d^{2}\left(x_{k}, F^{*}\right)} \int_{\frac{1}{64} d^{2}\left(x_{k}, F^{*}\right)}^{\frac{103}{64} d^{2}\left(x_{k}, F^{*}\right)} \gamma\left(B\left(x_{k}, 4 \sqrt{s}\right) d s\right. \\
& \lesssim\left(\sup _{z \in F}\left|T_{(4,4 d)}^{*} u(z)\right|^{2}\right) \sum_{k} \gamma\left(B\left(x_{k}, \frac{1}{2} \sqrt{103} d\left(x_{k}, F^{*}\right)\right)\right) \\
& \lesssim\left(\sup _{z \in F}\left|T_{(4,4 d)}^{*} u(z)\right|^{2}\right) \sum_{k} \gamma\left(B\left(x_{k}, d\left(x_{k}, F^{*}\right)\right)\right) \\
& \lesssim\left(\sup _{z \in F}\left|T_{(4,4 d)}^{*} u(y)\right|^{2}\right) \gamma\left(\complement F^{*}\right),
\end{aligned}
$$

where the second last step used the doubling property for admissible balls (recalling that $B\left(x_{k}, d\left(x_{k}, F^{*}\right)\right) \in \mathscr{B}_{2}$ ), and the last step used (4.6).

To estimate $I_{5}$, we proceed as we did for $I_{1}$. Writing $c:=c_{2 K, 3}$ we obtain

$$
\begin{aligned}
I_{5} & \lesssim \int_{\widetilde{B}_{3}^{\varepsilon}} \int_{F \cap B(y, 3 t)} \frac{1}{\gamma(B(y, t))}\left|e^{-t^{2} L} u(y)\right|^{2} d \gamma(z) d \gamma(y) \frac{d t}{t} \\
& \leq \int_{\mathbb{R}^{n}} \int_{m(y)}^{2 K m(y)} \int_{F} \frac{1_{B(y, 3 t)}(z)}{\gamma(B(y, t))}\left|e^{-t^{2} L} u(y)\right|^{2} d \gamma(z) \frac{d t}{t} d \gamma(y) \\
& \stackrel{(\mathrm{i})}{\leq} \int_{F} \int_{(1+3 c)^{-1} m(z)}^{c m(z)} \int_{B(z, 3 t)} \frac{1}{\gamma(B(y, t))}\left|e^{-t^{2} L} u(y)\right|^{2} d \gamma(y) \frac{d t}{t} d \gamma(z) \\
& \lesssim \int_{F}\left|T_{(2 K, c)}^{*} u(z)\right|^{2} d \gamma(z),
\end{aligned}
$$

where in step (i) we used that $m(y) \leq t \leq 2 K m(y)$ and $|y-z|<3 t$ imply $t \leq \mathrm{cm}(z)$ by Lemma 2.2(i), so $|y-z|<3 \mathrm{~cm}(z)$, and by an application of Lemma 2.2(ii) the latter implies $m(z) \leq(1+3 c) m(y) \leq(1+3 c) t$.

Finally we turn to $I_{6}$, which is treated as $I_{2}$. With $c=c_{2 K, 3}$ and $d=(1+3 c)^{-1}$ as in the previous estimate, and using Lemma 4.1 as in 
the estimate for $I_{2}$, we get

$$
\begin{aligned}
I_{6} & \lesssim \int_{F} \int_{d m(z)}^{c m(z)} \frac{1}{\gamma(B(z, 3 t))} \int_{B(z, 3 t)}\left|t \nabla e^{-t^{2} L} u(y)\right|^{2} d \gamma(y) \frac{d t}{t} d \gamma(z) \\
& =\frac{1}{2} \int_{F} \int_{d^{2} m(z)^{2}}^{c^{2} m(z)^{2}} \frac{1}{\gamma(B(z, 3 t))} \int_{B(z, 3 t)}\left|\nabla e^{-s L} u(y)\right|^{2} d \gamma(y) d s d \gamma(z) \\
& \lesssim \int_{F}(1+m(z)|z|)\left|T_{(A, a)}^{*} u(z)\right|^{2} d \gamma(z) \\
& \lesssim \int_{F}\left|T_{(A, a)}^{*} u(z)\right|^{2} d \gamma(z),
\end{aligned}
$$

for certain $A, a$ independent of $u, F$, and $\varepsilon$.

Combining all these estimates, we obtain six couples $\left(A^{(j)}, a^{(j)}\right)(j=$ $1, \ldots, 6)$, and, passing to the limit $\varepsilon \downarrow 0$, the following estimate, valid for arbitrary closed subsets $F \subseteq \mathbb{R}^{n}$ :

$$
\int_{F^{*}}\left|S_{a} u(x)\right|^{2} d \gamma(x)
$$

$$
\lesssim \sum_{j=1}^{6}\left(\left(\sup _{z \in F}\left|T_{\left(A^{(j)}, a^{(j)}\right)}^{*} u(z)\right|^{2}\right) \gamma\left(\mathcal{C} F^{*}\right)+\int_{F}\left|T_{\left(A^{(j)}, a^{(j)}\right)}^{*} u(z)\right|^{2} d \gamma(z)\right),
$$

with constants independent of $F$ and $u$.

To finish the proof, we consider the distribution functions

$$
\begin{aligned}
\gamma_{S_{a} u}(\sigma) & :=\gamma\left(\left\{x \in \mathbb{R}^{n}: S_{a} u(x)>\sigma\right\}\right), \\
\gamma_{T_{\left(A^{(j)}, a^{(j)}\right)} u}(\sigma) & :=\gamma\left(\left\{x \in \mathbb{R}^{n}: T_{\left(A^{(j)}, a^{(j)}\right)}^{*} u(x)>\sigma\right\}\right), \quad j=1, \ldots, 6 .
\end{aligned}
$$

We fix $\sigma>0$ for the moment, and apply (4.8) to the set

$$
F_{\sigma}:=\left\{z \in \mathbb{R}^{n}: T_{\left(A^{(j)}, a^{(j)}\right)}^{*} u(z) \leq \sigma, \quad j=1, \ldots, 6\right\},
$$

and claim that $\complement F_{\sigma}^{*} c \subseteq\left\{M_{\widetilde{K}}^{*}\left(1_{C F_{\sigma}}\right)>\frac{1}{2}\right\}$. Indeed, let $x \in \complement F_{\sigma}^{*}$ and fix $r \in(0, \widetilde{K} m(x)]$ such that $\gamma\left(B(x, r) \cap F_{\sigma}\right)<\frac{1}{2} \gamma(B(x, r))$. Then

$$
M_{\widetilde{K}}^{*}\left(1_{C_{F_{\sigma}}}\right)(x) \geq \frac{\gamma\left(B(x, r) \cap \complement F_{\sigma}\right)}{\gamma(B(x, r))}>\frac{1}{2},
$$

proving the claim.

Lemma 3.2 (with admissibility parameter $\widetilde{K}, \tau=\frac{1}{2}$, applied to the function $\left.1_{C_{F_{\sigma}}}\right)$ gives us $\gamma\left(\complement F_{\sigma}^{*}\right) \lesssim \gamma\left(\complement F_{\sigma}\right)$. Using this in combination 
with the definition of $F_{\sigma}$, for $j=1, \ldots, 6$ we obtain

$$
\begin{aligned}
\frac{1}{\sigma^{2}}\left(\sup _{z \in F_{\sigma}}\left|T_{A^{(j)}, a^{(j)}}^{*} u(z)\right|^{2}\right) \gamma\left(\complement F_{\sigma}^{*}\right) & \leq \gamma\left(\complement F_{\sigma}^{*}\right) \\
& \lesssim \gamma\left(\complement F_{\sigma}\right) \leq \sum_{k=1}^{6} \gamma\left(\left\{T_{\left(A^{(k)}, a^{(k)}\right)}^{*} u>\sigma\right\}\right) .
\end{aligned}
$$

Hence, from (4.8) we infer

$$
\begin{aligned}
\gamma_{S_{a} u}(\sigma) & \leq \gamma\left(F_{\sigma}^{*} \cap\left\{S_{a} u>\sigma\right\}\right)+\gamma\left(\complement F_{\sigma}^{*}\right) \\
& \lesssim \frac{1}{\sigma^{2}} \int_{F_{\sigma}^{*}}\left|S_{a} u(x)\right|^{2} d \gamma(x)+\gamma\left(\complement F_{\sigma}\right) \\
& \lesssim \sum_{j=1}^{6}\left[\gamma_{\left.T_{\left(A^{(j)}, a\right.}^{*}(j)\right)} u(\sigma)+\frac{1}{\sigma^{2}} \int_{F_{\sigma}}\left|T_{\left(A^{(j)}, a^{(j)}\right)}^{*} u(z)\right|^{2} d \gamma(z)\right] \\
& \lesssim \sum_{j=1}^{6}\left[\gamma_{\left.T_{\left(A^{(j)}, a\right.}^{(j)}\right)} u(\sigma)+\frac{1}{\sigma^{2}} \int_{0}^{\sigma} t \gamma_{\left.T_{\left(A^{(j)}, a\right.}^{(j)}\right)} u(t) d t\right]
\end{aligned}
$$

Integrating over $\sigma$ and noting that

$$
\begin{aligned}
\int_{0}^{\infty} \frac{1}{\sigma^{2}} \int_{0}^{\sigma} t \gamma_{T_{(A(j), a}^{*}(j)}{ }^{u}(t) d t d \sigma & =\int_{0}^{\infty} t \gamma_{\left.T_{\left(A^{(j)}, a\right.}^{*}(j)\right)} u(t) \int_{t}^{\infty} \frac{1}{\sigma^{2}} d \sigma d t \\
& =\int_{0}^{\infty} \gamma_{T_{\left(A^{(j)}, a^{(j)}\right)}^{*}}(t) d t=\left\|T_{\left(A^{(j)}, a^{(j)}\right)}^{*} u\right\|_{L^{1}(\gamma)},
\end{aligned}
$$

we get, by Theorem 3.1 and with $a^{(j) \prime}$ as in the statement of that theorem,

$$
\begin{aligned}
\left\|S_{a} u\right\|_{L^{1}(\gamma)} & \lesssim \sum_{j=1}^{6}\left\|T_{\left(A^{(j)}, a^{(j)}\right)}^{*} u\right\|_{L^{1}(\gamma)} \\
& \lesssim \sum_{j=1}^{6}\left\|T_{\left(1, a^{(j)}\right)^{\prime}}^{*} u\right\|_{L^{1}(\gamma)} \leq 6\left\|T_{\left(1, a^{\prime}\right)}^{*} u\right\|_{L^{1}(\gamma)},
\end{aligned}
$$

where $a^{\prime}=\max _{j=1, \ldots, 6} a^{(j) \prime}$ 
Remark 4.2. In $[\mathbf{8}]$ the cones

$$
\widetilde{\Gamma}_{x}^{(A, a)}(\gamma):=\left\{(y, t) \in \mathbb{R}^{n} \times(0, \infty):|y-x|<A t \text { and } t<a m(y)\right\}
$$

are used implicitly. Set $a^{\prime}:=c_{a, A}$. In view of the inclusions

$$
\Gamma_{x}^{(A, a)}(\gamma) \subseteq \widetilde{\Gamma}_{x}^{\left(A, a^{\prime}\right)}(\gamma), \quad \widetilde{\Gamma}_{x}^{(A, a)}(\gamma) \subseteq \Gamma_{x}^{\left(A, a^{\prime}\right)}(\gamma),
$$

the corresponding functions $\widetilde{S}$ and $\widetilde{T}^{*}$ satisfy the pointwise bounds

$$
\widetilde{S}_{a} u(x) \lesssim S_{a^{\prime}} u(x), \quad S_{a} u(x) \lesssim \widetilde{S}_{a^{\prime}} u(x)
$$

and

$$
\widetilde{T}_{(A, a)}^{*} u(x) \lesssim T_{\left(A, a^{\prime}\right)}^{*} u(x), \quad T_{(A, a)}^{*} u(x) \lesssim \widetilde{T}_{\left(A, a^{\prime}\right)}^{*} u(x) .
$$

In particular, Theorem 1.1 remains valid if we replace $S$ and $T^{*}$ by $\widetilde{S}$ and $\widetilde{T}^{*}$. Remark also that $[\mathbf{8}$, Theorem 3.8] gives a change of aperture formula for tent spaces that implies an analogue of Theorem 3.1 for the square function $\widetilde{S}$ for $A, A^{\prime}>1$.

Remark 4.3. We conclude with a few words on reverse inequalities, i.e., controls of the maximal function by the square function. In the euclidean case, such inequalities are generally proven via atomic decompositions, usually going through tent spaces. We have developed, in [8], the gaussian analogues of these spaces and their atomic decomposition. However, to deduce a reverse inequality, we would then need an adequate analogue of the Calderón reproducing formula (analogues exist, but do not seem to be appropriate), and such a formula is involving all $t \in(0, \infty)$ rather than just $t \in(0, a m(x))$. A complete Hardy space theory is thus likely to require an understanding of the "non-admissible" parts of objects such as $T^{*} u, S u$, or Mauceri-Meda's atoms (i.e. the part corresponding to the scales $t \in(\operatorname{am}(x), \infty)$, for which balls are not admissible), or a technique that allows one to avoid such non-admissible part in arguments involving Calderón reproducing formulae. This is the subject of some of our on-going investigations.

\section{References}

[1] P. Auscher, A. McIntosh, and E. Russ, Hardy spaces of differential forms on Riemannian manifolds, J. Geom. Anal. 18(1) (2008), 192-248.

[2] P. Auscher And E. Russ, Hardy spaces and divergence operators on strongly Lipschitz domains of $\mathbb{R}^{n}$, J. Funct. Anal. 201(1) (2003), 148-184. 
[3] R. R. Coifman, Y. Meyer, and E. M. Stein, Some new function spaces and their applications to harmonic analysis, J. Funct. Anal. 62(2) (1985), 304-335.

[4] B. E. J. Dahlberg, C. E. Kenig, J. Pipher, and G. C. VerСнотA, Area integral estimates for higher order elliptic equations and systems, Ann. Inst. Fourier (Grenoble) 47(5) (1997), $1425-1461$.

[5] C. Fefferman and E. M. Stein, $H^{p}$ spaces of several variables, Acta Math. 129(3-4) (1972), 137-193.

[6] L. Forzani, R. Scotto, and W. Urbina, Riesz and Bessel potentials, the $g^{k}$ functions and an area function for the Gaussian measure $\gamma$, Rev. Un. Mat. Argentina 42(1) (2000), 17-37 (2001).

[7] S. Hofmann and S. MaYboroda, Hardy and BMO spaces associated to divergence form elliptic operators, Math. Ann. 344(1) (2009), 37-116.

[8] J. Maas, J. van Neerven, and P. Portal, Whitney coverings and the tent spaces $T^{1, q}(\gamma)$ for the Gaussian measure, Ark. Mat. (to appear); arXiv:1002.4911.

[9] G. Mauceri and S. Meda, BMO and $H^{1}$ for the Ornstein-Uhlenbeck operator, J. Funct. Anal. 252(1) (2007), 278-313.

[10] G. Mauceri, S. Meda, And P. SJÖGren, Endpoint estimates for first-order Riesz transforms associated to the Ornstein-Uhlenbeck operator; arXiv:1002.1240.

[11] B. Muckenhoupt, Hermite conjugate expansions, Trans. Amer. Math. Soc. 139 (1969), 243-260.

[12] E. Pineda and W. Urbina R., Non tangential convergence for the Ornstein-Uhlenbeck semigroup, Divulg. Mat. 16(1) (2008), 107-124.

[13] P. SJÖGREn, Operators associated with the Hermite semigroup - a survey, in: "Proceedings of the conference dedicated to Professor Miguel de Guzmán" (El Escorial, 1996), J. Fourier Anal. Appl. 3, Special Issue (1997), 813-823.

[14] E. M. Stein, "Singular integrals and differentiability properties of functions", Mathematical Series 30, Princeton University Press, Princeton, N.J., 1970. 
Jan Maas:

Institute for Applied Mathematics

University of Bonn

Endenicher Allee 60

53115 Bonn

Germany

E-mail address: maas@iam.uni-bonn.de

Jan van Neerven:

Delft Institute of Applied Mathematics

Delft University of Technology

P.O. Box 5031

2600 GA Delft

The Netherlands

E-mail address: J.M.A.M.vanNeerven@tudelft.nl

Pierre Portal:

Université Lille 1

Laboratoire Paul Painlevé

59655 Villeneuve d'Ascq

France

E-mail address: pierre.portal@math.univ-lille1.fr

Primera versió rebuda el 6 d'abril de 2010, darrera versió rebuda el 28 de gener de 2011. 